Le colporteur de Proserpine : un catalogue satirique dans Les Promenades d'Eustache Le Noble

\title{
Helwi Blom
}

Author's version of the chapter published in Anne-Pascale Pouey-Mounou and Paul J. Smith (eds.), Early Modern Catalogues of Imaginary Books. A Scholarly Anthology Leiden/Boston: Brill, 2020), pp. 299-319. Publication Date: 28-11-2019.

https://doi.org/10.1163/9789004413658

Parmi les nombreuses publications que nous a laissées le polygraphe français Eustache [II] Le Noble (1643-1711) se trouve un texte intitulé significativement Le Colporteur de Proserpine. ${ }^{1}$ Ce livret fait partie d'un ouvrage périodique publié sous le titre général de L'École du monde nouvelle ou Les Promenades de Mr. Le Noble et parut probablement pour la première fois vers la mi-avril $1699 .^{2}$

À cette époque-là Le Noble vivait essentiellement de sa plume. Issu d'une famille noble parvenue à la magistrature de Troyes et ayant fait des études brillantes, il avait épousé en 1648 la fille d'un riche marchand troyen qui lui avait apporté une large dot. Après avoir exercé pendant quelques années comme avocat, Le Noble devint en 1673 conseiller et procureur général du roi en la cour du Parlement de Metz. Tout semblait le destiner à une belle carrière et à une vie en toute aisance, mais suite à une série de scandales et de démêlés avec la justice, Le Noble fut obligé de vendre sa charge et vers 1686, il ne lui restait pas d'autre moyen de subsistance que sa plume. Heureusement il savait bien s'en servir. Grâce à ses pamphlets politiques visant à soutenir la politique étrangère de Louis XIV, il acquit très vite une certaine notoriété. À partir de 1694, la part de la politique et de l'histoire dans ses ouvrages commença cependant à diminuer au profit d'une littérature de fiction divertissante et moralisante dont la majeure partie vit le jour sous forme de publications périodiques de quelques dizaines de pages.

\footnotetext{
${ }^{1}$ Je remercie vivement Katell Lavéant pour sa relecture soignée de cet article.

${ }^{2}$ Les 25 promenades composant la série ont vu le jour entre janvier et décembre 1699 à raison d'environ deux livraisons par mois. Le colporteur de Proserpine en constitue le septième numéro. L'original est perdu. L'édition la plus ancienne conservée de ce texte est une réimpression de 1700 par les héritiers d'Antoine Schelte à Amsterdam.
} 
Bien que Le Noble n'ait pas fait partie des milieux littéraires officiels et qu'il n'ait pas participé aux grands débats qui divisaient le Parnasse français vers la fin du XVII ${ }^{\mathrm{e}}$ siècle, il prenait sa réputation d'homme de lettres très au sérieux et pas seulement parce qu'il se souciait de la vente de ses œuvres. Il se faisait gloire de chanter les louanges des grands auteurs classiques et de mettre ses contemporains en garde contre des productions littéraires de mauvais goût ou de médiocre qualité. Le Noble était en effet d'avis qu'en tant que rejeton d'une vieille famille noble élevé dans la tradition humaniste, il était très bien placé pour jouer à la fois le rôle d'écrivain satirique, d'historien et d'éducateur mondain. C'est pourquoi, dans ses œuvres, il a amplement critiqué des personnes dont les actes avaient suscité son indignation. Il a par exemple dénoncé sur tous les tons les idées et les pratiques malsaines des adversaires politiques de Louis XIV et il a également crié au scandale à propos de certains abus sociaux. Mais Le Noble n'a pas résisté non plus à la tentation de noircir des hommes et des femmes contre lesquels il avait surtout des rancunes personnelles. ${ }^{3}$

Un des instruments dont l'ancien procureur s'est servi pour émettre des critiques est la liste de livres imaginaires, genre littéraire dont on attribue généralement l'invention à François Rabelais. Par exemple, dans la série des pamphlets politiques ou de pasquinades qui parurent sous le titre de La Pierre de touche politique, Le Noble a publié en janvier 1690 La Bibliothèque $d u$ roi Guillemot, texte donnant un catalogue de titres fictifs visant à critiquer et à ridiculiser le stathouder néerlandais Guillaume [III] d'Orange. Celui-ci était devenu roi d'Angleterre après la Glorieuse Révolution de 1688 et figurait parmi les plus importants adversaires de Louis XIV. La Bibliothèque du roi Guillemot fait allusion à une relation de la visite que le nouveau roi anglais avait rendue à une des universités du pays et Le Noble décrit à son tour le 'principal incident du Régal' que Guillaume y aurait reçu, à savoir un tour de la bibliothèque. ${ }^{4}$ Sur une des tablettes de l'établissement auraient été posés plusieurs 'nouveaux livres curieux' portant sur les vertus et les actions héroïques du monarque, composés en son honneur par le personnel

\footnotetext{
${ }^{3}$ Sur la vie et les œuvres de Le Noble, voir Hourcade Ph., Entre Pic et Rétif: Eustache le Noble (1632-1711) (Paris: 1990).

${ }^{4}$ Selon Hourcade il s'agit de l'Université d'Oxford. Hourcade, Entre Pic et Rétif 242. Nous n'avons pas trouvé de sources faisant mention d'une visite du roi Guillaume à l'Université d'Oxford en 1689. The London Gazette du 10 octobre 1689 contient bien une relation d'une visite royale présentant des éléments qui correspondent à la description de Le Noble, mais cet événement a eu lieu à Cambridge. Cf. Malcolm J.P., Miscellaneous Anecdotes illustrative of the Manners and History of Europe during the Reigns of Charles II, James, II, William III and Q. Anne (London: 1811) 186-189.
} 
de l'université et qu'on lui offrait en cadeau. Suit une liste de titres et de 'sommaires' inventés par Le Noble, qui se lit comme une attaque incisive contre 'l'usurpateur' et ses partisans. ${ }^{5}$ Une des livraisons des Nouveaux entretiens politiques (1702-1709), intitulée La Bibliothèque de Salamanque, contient également un catalogue satirique composé par le Noble dans un but politique. $^{6}$

Le catalogue fictif que Le Noble fit publier en avril 1699 dans sa Septième Promenade diffère à plusieurs égards des deux listes de livres satiriques citées. Ces dernières ont été composées en un temps de guerre, tandis que Les Promenades datent d'une époque où la France connaissait une paix relative sur le plan international. Cette différence a eu des conséquences pour le fond et la forme des listes en question. La Bibliothèque du roi Guillemot et La Bibliothèque de Salamanque sont des pamphlets écrits sous la forme d'un dialogue entre deux personnages qui commentent les nouvelles de la guerre et les derniers développements politiques. Si ces dialogues sont ornés ici ou là par des textes relevant d'autres genres littéraires, comme des fables, des poèmes ou des nouvelles, la discussion politique en reste le composant essentiel. Le Colporteur de Proserpine est également un petit ouvrage écrit sous la forme d'un entretien, mais dans Les Promenades, on rencontre chaque fois quatre interlocuteurs, qui se retrouvent dans le Jardin des Tuileries. Leurs conversations ne portent pas sur la politique, mais sur les gens qui passent et sur des faits divers qui donnent parfois lieu à des réflexions plus générales d'ordre scientifique, social ou moral. L'entretien proprement dit s'y efface de plus en plus au profit d'autres formes artistiques, de sorte que celles-ci et notamment les nouvelles en deviennent finalement la matière principale.

\section{Claude Barbin, colporteur infernal}

Le Colporteur de Proserpine s'ouvre sur une rencontre des quatre amis avec un jeune auteur de pièces dramatiques, ce qui les amène à discuter de ce que le narrateur appelle 'les causes de la décadence honteuse du Téatre'. Les amis en viennent ensuite au sujet principal de leur entretien, le décès d'un personnage connu dans le monde littéraire de l'époque et qui avait le privilège

\footnotetext{
${ }^{5}$ [Le Noble Eustache], La Pierre de touche politique. Janvier 1690. La Bibliotheque du roi Guillemot. Sixième dialogue. Jouxte la copie imprimée (London, Jean Benn: 1690) 11-46. À la p. 14, Le Noble fait d'ailleurs explicitement référence à Rabelais en faisant remarquer à un des interlocuteurs que 'la Bibliotéque de Saint Victor, inventoriée par le visionnaire Rabelais, n'a rien qui aproche de' cette collection de livres offerts au roi Guillaume.

${ }^{6}$ Il s'agit de la 76 ${ }^{\mathrm{e}}$ livraison, d'août 1708. Paris, BnF, 8-LC2-58.
} 
douteux d'avoir attiré sur lui le regard critique, sinon jaloux, de Le Noble: le libraire Claude Barbin, décédé le 24 décembre 1698. Dans le texte, il est évoqué sous le nom du 'vieux marquis de Parangon' et de 'Barbinelli'.

Barbin était de son vivant un libraire célèbre, spécialisé dans l'édition d'ouvrages appréciés à la Cour et dans les milieux mondains. Il s'était entre autres bâti une belle réputation d'éditeur de petits ouvrages à la mode, comme le Mercure galant, une sorte de revue mondaine, et toutes sortes de contes et de nouvelles historiques ou galantes. Une large partie de ces livrets étaient composés par des auteurs féminins et les femmes prenaient encore la première place parmi leurs lecteurs. Le fait que Barbin avait fait un grand débit d'ouvrages éphémères peu sérieux, mais très populaires, lui avait valu des critiques sévères. On lui reprochait entre autres de faire des choix éditoriaux motivés par des considérations commerciales plutôt que par la qualité des œuvres retenues. ${ }^{7}$ Le Noble, dont les œuvres ne furent jamais éditées par Barbin, se trouvait parmi ceux qui méprisaient les nouvelles galantes et les autres petits livres à la mode. En 1690, notre auteur s'était déjà rangé dans le camp des détracteurs du libraire en remarquant à propos d'un certain sujet que 'quelque Pensionnaire de Barbin' y trouverait certainement de l'inspiration pour une nouvelle historique. ${ }^{8}$ Quelques années plus tard, dans son École du monde, il s'en prit de nouveau à ce qu'il appelait les 'colifichets de Barbin'. ${ }^{9}$ Il semblerait même que ce soit Le Noble qui ait inventé le mot de 'barbinades', terme dérivé du nom du libraire et utilisé au XVIII ${ }^{\mathrm{e}}$ siècle pour désigner de façon dédaigneuse le genre de productions légères dont Barbin avait en quelque sorte été le spécialiste. ${ }^{10}$

Puisant dans une veine satirique de longue date, exploitée sous différentes formes tout au long du XVII ${ }^{\mathrm{e}}$ siècle, entre autres par Le Noble lui-même, l'auteur du donne dans sa Septième

\footnotetext{
${ }^{7}$ Voir par exemple la lettre de Jean-Baptiste Dubos à Pierre Bayle du 28 mai 1686: 'Défiez-vous des livres imprimés chez Barbin; c'est merveille quand il y en a quelqu'un de bon. Autrefois la meilleure pièce de sa boutique était sa femme'. Citée dans Reed G.E, Claude Barbin, libraire de Paris sous le règne de Louis XIV (Geneva-Paris: 1974) 17.

${ }^{8}$ La Bibliothèque du roi Guillemot 46.

${ }^{9}$ Les Oeuvres de $M^{r}$ Le Noble, tome IV (Paris, Pierre Ribou: 1718) 16.

${ }^{10}$ Le mot est attesté dans le Dictionnaire de Trévoux de 1743. Il n'apparaît pas dans l'édition de 1721. La plus ancienne mention de ce terme que nous ayons repérée se trouve dans le $20^{\mathrm{e}}$ entretien de L'École du monde, publié vers la fin de l'année 1695. Le Noble y fait dire à un des interlocuteurs qu'une certaine dame 'ne pourroit pas faire la difference des ouvrages de Despreaux ou de l'Auteur des Pasquinades, d'avec les Barbinades où le Mercure Galand'. L'autre interlocuteur lui demande alors ce qu'il entend par 'Barbinades', et le premier explique qu'il donne ce nom 'à ces nombreux Colifichets de petits Livres qui ne servent qu'à faire perdre inutilement du temps, \& aprés la lecture desquels on se trouve l'esprit aussi peu rempli que si l'on n'avoit rien lû, \& qui n'ont pas laissé d'enrichir nôtre ami Barbin.' Les Oeuvres de Mr Le Noble, tome IV 150.
} 
Promenade une relation des rencontres et des occupations de feu Barbin après son passage dans l'autre monde. Pour cela, il a fait le choix de construire une histoire enchâssée dans le texte de l'entretien, que Le Noble présente sous la forme d'un mémoire anonyme, trouvé par un des interlocuteurs et lu par lui à ses amis réunis dans le Jardin des Tuileries. Le texte du mémoire, intitulé Le Colporteur de Proserpine, nouvelle des enfers, constitue la partie la plus importante de l'entretien auquel il a donné son titre. En enfer, Barbin retrouve les grands auteurs de son catalogue et il les met au courant du devenir de leurs créations littéraires en lançant quelques commentaires piquants sur la qualité de leurs ouvrages et sur l'état du Parnasse français. Ensuite, le nouveau venu est reçu par la reine des enfers, qui fait du libraire mondain le fournisseur de la cour infernale. Le résumé de l'entretien de Proserpine avec Barbin et les raisons que donne le narrateur pour expliquer l'intérêt de la grande dame pour l'ancien libraire font écho aux moqueries qui se faisaient entendre dans la société de l'époque à propos du fonds de Barbin, de ses ambitions et de son public. Le Noble a poussé son jeu de parallèles jusqu'à situer la boutique que Barbin est autorisé à ouvrir à l'entrée du Palais de Justice. Quant aux livres qu'il va étaler, c'est évidemment en décrivant les titres du catalogue de Barbin que l'auteur a pensé faire son coup. Il combine alors le procédé satirique et facétieux des nouvelles de l'autre monde avec celui de la liste de livres imaginaires. C'est en effet un instrument plus qu'approprié pour s'attaquer à un libraire.

Comme on s'y pourrait attendre, les livres que Barbin propose en enfer sont des bagatelles. Mais ce ne sont pas des nouveautés écrites par des auteurs mondains désireux de répondre au goût du beau monde, comme le libraire en avait vendu à Paris. Il s'agit d'un genre de petits livres beaucoup moins estimé: à la demande de Barbin, le dieu Mercure passe chez l'éditeur troyen Oudot pour se procurer 'tous les plus beaux enfans bleus' qu'il y verra. Dans la France de l'époque, l'expression 'enfants bleus' faisait référence aux orphelins ou aux enfants abandonnés de l'Hôpital de la Trinité, qui portaient un uniforme de drap bleu. Le Noble a associé l'image de ces pauvres enfants vêtus de bleu aux livres vendus par Oudot pour souligner les correspondances entre les deux sur le plan de leur habillement et de leur position socioculturelle, pour ainsi dire. La collection des Oudot et de leurs confrères troyens se composait principalement d'ouvrages produits à moindre coût et brochés d'une simple couverture en papier bleu. Il s'agissait d'éditions à grande diffusion de titres qui étaient tombés dans le domaine public. Les réimpressions de la littérature chevaleresque du Moyen Âge, qui avaient autrefois fait les délices de l'aristocratie et de la haute bourgeoisie, y étaient bien représentées, mais on y trouvait aussi des éditions bon marché de textes littéraires 
contemporains (pièces de théâtre, littérature facétieuse, contes). Il y avait également toute une gamme de littérature édifiante et nombre d'ouvrages à fins utilitaires: almanachs, manuels de civilité, livres de recettes, etc. Ces productions sortaient alors en grande quantité de la ville champenoise pour être vendues par des colporteurs et par des libraires correspondants, entre autres à la capitale. Elles avaient gagné une certaine renommée et le public avait commencé à les surnommer 'la Bibliothèque bleue'. Les 'livres bleus' étaient destinés à un public divers, mais pendant le dernier tiers du XVII ${ }^{\mathrm{e}}$ siècle, ils étaient surtout associés aux enfants et aux lecteurs non cultivés des classes modestes qui n'avaient ni les moyens ni les capacités culturelles pour acheter et lire les nouveautés littéraires ou des ouvrages savants. Il semble d'ailleurs que vers la fin du siècle, il y ait eu un certain regain d'intérêt pour les vieux romans diffusés par la Bibliothèque bleue dans les milieux cultivés, mais cela n'empêche pas que les références du public privilégié aux 'livres bleus’ étaient généralement marquées par un profond mépris pour le contenu de ces publications et pour leurs lecteurs. ${ }^{11}$

Étant originaire de Troyes, Le Noble avait sans aucun doute lui-même feuilleté plusieurs de ces modestes imprimés, tout au moins dans sa jeunesse, avec ses camarades du collège de 1'Oratoire. Les romans de chevalerie dont Nicolas [II] Oudot avait alors fait paraitre des éditions bon marché illustrées de vieux bois gravés - souvent copiés sur les images ayant servi dans les éditions du $\mathrm{XVI}^{\mathrm{e}}$ siècle - doivent avoir captivé ces jeunes collégiens d'une façon comparable à celle qu'évoque Charles Sorel en décrivant les passetemps du jeune Francion. ${ }^{12}$ De ce point de vue il n'est pas étonnant que la liste des 'livres bleus' que Mercure rapporte de la boutique des Oudot et dont le mémoire cite une dizaine de titres contienne presque uniquement de vieux romans de chevalerie.

Le caractère satirique du catalogue fictif présenté dans Le Colporteur de Proserpine réside donc premièrement dans le fait que Le Noble a transformé Claude Barbin, le libraire parisien huppé, en un colporteur de livres de second rang et qu'il a rempli sa boutique infernale de livres qui étaient, de l'avis de l'auteur du mémoire, aussi curieux que ceux que Barbin vendait sur terre. En dressant un tel catalogue d'anciens romans chevaleresques, Le Noble s'est

\footnotetext{
${ }^{11}$ Voir à ce sujet notre thèse (encore inédite) Blom H., «Vieux Romans » et « Grand Siècle »; éditions et réceptions de la littérature chevaleresque médiévale dans la France du dix-septième siècle (Université d'Utrecht: 2012). Le Noble n'était d'ailleurs pas le premier à faire un parallèle entre les enfants de la Trinité et les livres de la Bibliothèque bleue. Un autre catalogue fictif du XVII siècle, celui des livres de Mythophilacte dans le Roman bourgeois (1666) d'Antoine Furetière, avait déjà comparé les livres en papier bleu à ces pauvres orphelins. Furetière Antoine, Le Roman bourgeois (Nancy, Jean-Baptiste Cusson: 1713) 300.

${ }^{12}$ Cf. Sorel Charles, Histoire comique de Francion, éd. Y. Giraud (Paris: 1979) 171-173.
} 
rangé dans la lignée des auteurs satiriques et burlesques qui s'étaient amusés avant lui à donner des énumérations plus ou moins facétieuses de vieux romans et de livres populaires. Dans la littérature du Grand Siècle, nous avons ainsi repéré plusieurs descriptions de bibliothèques ou de boutiques imaginaires dont les vieux romans de chevalerie constituent le noyau dur. Pendant la première moitié du siècle, la plupart de ces listes avaient déjà pour but de critiquer la frivolité et le goût littéraire de ceux qui possédaient ce genre de livres, mais elles n'en étaient pas moins le reflet du contenu de mainte bibliothèque contemporaine. À partir des années 1650, quand l'accès au livre s'élargit grâce à l'alphabétisation et aux éditions modestes de la Bibliothèque bleue, dont le prix allait encore en s'amoindrissant, la critique de ce genre de listes de romans de chevalerie et d'autres livres populaires visait plutôt le fait qu'il s'agissait de livres de peu de qualité, destinés à un public modeste et inculte. ${ }^{13}$

\section{Titres et sous-titres satiriques}

La satire se joue cependant à plus d'un niveau dans ce catalogue fictif de Le Noble. Si la plupart des titres qui figurent sur la liste des ouvrages achetés par Mercure sont des titres de livres qui se trouvaient en 1699 effectivement chez les frères Jean [III] en Jacques [II] Oudot à Troyes, Le Noble les a pourvus de sous-titres et de données bibliographiques nouveaux, qui n'ont rien à faire avec le contenu des 'livres bleus' en question. Il a même ajouté quelques titres complètement imaginaires au catalogue des Oudot.

À la première lecture de la liste de Mercure, le sens satirique des sous-titres et des données bibliographiques inventés par Le Noble nous a presque entièrement échappé. Il n'y avait en tout cas guère de points de repère pour relier les livres cités aux affaires du libraire Barbin, sauf pour le dernier titre, Mélusine, ou la Vieille fardée, une historiette que Barbin offrit en premier à la reine Proserpine et que le narrateur appelle 'l'ornement principal' de sa boutique. Le texte de cette historiette est intégralement transcrit dans Le Colporteur de Proserpine et constitue donc une nouvelle dans une nouvelle. En 1699, ce titre de Mélusine, ou la Vieille fardée a probablement réjoui les lecteurs de Le Noble. L'Histoire de Mélusine était en effet un des titres à succès de la Bibliothèque bleue de Troyes. Le texte des 'éditions bleues' était dérivé d'un roman de Mélusine en prose composé en 1393 par Jean d'Arras. Les éditeurs du $\mathrm{XVI}^{\mathrm{e}}$ siècle en avaient tiré deux romans distincts, celui de Mélusine et un autre roman racontant les aventures de son fils Geoffroy à la Grand'dent. Tout au long du XVII ${ }^{\mathrm{e}}$ siècle, le

\footnotetext{
${ }^{13}$ Cf. Blom, « Vieux Romans » notamment 314-320.
} 
roman médiéval sur la dame-fée Mélusine avait connu des réimpressions dans la Bibliothèque bleue troyenne. Or, en janvier 1698, en plein milieu de la vogue des contes des fées que Charles Perrault avait si habilement su remettre à la mode, notamment à la Cour, le libraire Claude Barbin avait publié une adaptation du roman médiéval de Mélusine, mise à jour par François Nodot et dédiée à Mademoiselle. Cette nouvelle Mélusine avait récolté un succès considérable auprès des lecteurs mondains. Nodot avait transformé la fée 'maternelle et défricheuse' de la légende en une dame galante que l'on pouvait croiser dans les salons mondains contemporains. En d'autres termes : il lui avait mis du fard Louis XIV. ${ }^{14}$ Une historiette de la boutique d'un Barbinelli intitulée Mélusine ou la Vieille fardée doit certainement avoir remis à l'esprit des lecteurs de Le Noble cette adaptation moderne du vieux roman de Mélusine réalisée par Nodot. La référence malveillante à l'Histoire de Mélusine se limite d'ailleurs au titre de l'historiette, car la trame et le style ne ressemblent en rien à l'histoire contenue dans le 'livre bleu'. Il s'agit en fait d'une nouvelle originale à peu près conforme aux normes du genre tel qu'il se développa à la fin du XVII ${ }^{\mathrm{e}}$ siècle.

L'avant-dernier titre du le catalogue de Mercure est apparenté à l'Histoire de Mélusine. Il s'agit du deuxième roman tiré au $\mathrm{XVI}^{\mathrm{e}}$ siècle du roman médiéval de Jean d'Arras, à savoir Les Gestes et faits de Geoffroy à la grand'dent, consacré aux exploits d'un des fils de Mélusine. Dans le premier tiers du XVII ${ }^{\mathrm{e}}$ siècle, ce roman était sorti plusieurs fois des presses des imprimeurs troyens, mais, pour autant que nous sachions, il n'était plus imprimé à l'époque qui nous intéresse. Le succès du remaniement de l'Histoire de Mélusine avait encouragé François Nodot à en adapter aussi la suite. Le 23 janvier 1699, il avait obtenu un privilège royal pour imprimer son adaptation, mais l'ouvrage ne parut qu'en 1700 à la suite de la réimpression de l'Histoire de Mélusine par la veuve de Claude Barbin. Il paraît alors peu probable que Le Noble fasse ici allusion à une publication de Barbin. Le sous-titre semble en effet nous éloigner de Barbin et de ses livres pour nous faire entrer au Palais de la Cité: Geofroi à la Grand dent, ou le Plaideur Béat [...]. ${ }^{15}$ Au lieu des faits et gestes d'un chevalier médiéval, ce titre évoque de manière satirique les affaires d'un certain Théogon qui épuise sa fortune en menant un procès. Le nom de Théogon est emprunté au grec et signifie probablement 'fils de dieu'. Le Noble avait eu une formation humaniste et faisait souvent allusion à des auteurs et à des œuvres de

\footnotetext{
${ }^{14}$ Cf. Blom, «Vieux Romans » 278-280.

${ }^{15}$ Pour les titres complets des ouvrages cités sur la liste de Mercure, voir la transcription partielle du catalogue en annexe.
} 
l'Antiquité, mais ici il semble s'agir d'un nom inventé. Au vu l'adjectif 'béat' et d'une remarque sur la 'pieuse destination des fondateurs' à la fin du titre, on pourrait penser à un ecclésiastique ou en tout cas à un homme religieux, mais nous n'avons pas encore identifié la personne que Le Noble avait en vue. Le thème du plaideur insensé gaspillant son argent dans un procès éternel qui lui coûte plus qu'il ne lui rapporte revient en tout cas régulièrement dans L'École du monde et dans les autres écrits moralistes de l'auteur, car l'ancien procureur a condamné à plusieurs reprises ces officiers de la loi avides qui poussaient leurs clients à poursuivre des procès inutiles. Non sans une certaine hypocrisie d'ailleurs, mais cela ne l'empêchait pas de donner sa critique comme une leçon morale à prendre à cœur.

\section{Une autre bête noire}

Le troisième titre sur la liste est celui des Quatre filles Aymon, référence ludique à l'histoire médiévale des Quatre fils Aymon. Ce roman chevaleresque était un des best-sellers du répertoire des éditeurs de la Bibliothèque bleue. Le titre composé par Le Noble est à première vue une véritable énigme pour le lecteur moderne: Les quatre filles Aymon, ou les Enfans trouvez. Petit Livre [...] sur la merveilleuse \& sage conduite du fameux Cornificius, \& comment par les conseils d'un Maître Yves aussi cornu que lui, \& à force de remuer la corne d'abondance dont il est authentiquement pourvû, il a eu le bonheur d'en faire sortir deux jolies petites Nymphes [...]. L'auteur veut apparemment ridiculiser un homme qui d'une manière ou d'une autre serait devenu père de deux filles. Mais qui est ce Cornificius? Le Noble réfère-t-il ici au rhéteur et grammairien romain du premier siècle? Qu'y a-t-il de spécial ou d'amusant à avoir deux filles? Pourquoi est-il question d'“enfants trouvés'? Un document juridique de 1700 nous a aidée à élucider ces points. Il s'agit d'un plaidoyer, imprimé pour servir dans un procès opposant un certain Louis Semitte, marchand épicier à Paris, à son épouse Marie-Gabrielle Perreau, qu'il avait accusée d'adultère. ${ }^{16}$ Semitte s'y plaint entre autres de Le Noble, parce que celui-ci l'aurait insulté plusieurs fois dans ses publications, notamment en faisant de lui le protagoniste de ce livre des Quatre filles Aymon cité dans la Septième Promenade.

Pour comprendre cette accusation, il faut savoir que Le Noble était impliqué dans le procès que l'épicier avait intenté contre son épouse et dont le début remontait à 1692. En 1693,

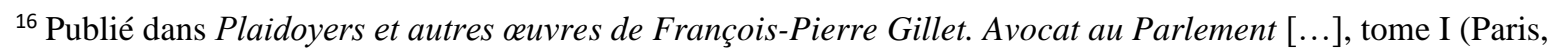
Gabriel Martin: 1718) 229-279. Il existe une version légèrement différente du même factum signée par maître Aligier en tant que procureur de Semitte (Paris, BnF, 4-FM-29939).
} 
Le Noble avait rencontré la belle dame en prison où il purgeait lui-même une peine pour faux en écriture. Le Noble et la belle épicière nouèrent une relation amoureuse et l'auteur mit ses talents juridiques et rhétoriques au service de sa maîtresse. En fait, l'histoire que l'on peut partiellement reconstituer à partir du factum et d'autres documents historiques que nous avons recueillis a tous les ingrédients d'un feuilleton télévisé.

En 1694, Marie-Gabrielle Perreau, qui avait été condamnée pour adultère et enfermée dans un couvent, accoucha en secret d'un enfant de Le Noble. Le bébé fut baptisé du nom du Chevalier de St. George, un des titres que portait Le Noble. En décembre 1694, Marie-Gabrielle s'évada du couvent et, quelques mois plus tard, en mai 1695, Le Noble lui-même s'échappa de la Conciergerie. Les deux amants fugitifs se retrouvèrent et vécurent ensemble jusqu'à la capture de Le Noble en avril 1697. En août 1696, Marie-Gabrielle mit au monde une fille appelée Louise-Catherine. La petite fut exposée en mai 1697 et accueillie ensuite à l'Hôpital des Enfants-Trouvés. En mars 1698 Le Noble sortit de nouveau de prison, cette fois-ci de façon légale, mais sa réunion avec la belle épicière fut de courte durée; en octobre de la même année, Marie-Gabrielle, qui attendait de nouveau un enfant, fut arrêtée et enfermée à la Conciergerie.

Pendant toutes ces années, le procès opposant la Perreau à son époux légitime connut de nombreuses péripéties, notamment grâce à la ténacité et à la créativité de Le Noble, qui usa sa plume à écrire des requêtes, des défenses, des libelles et même de fausses lettres pour sa bien-aimée. Il va de soi qu'il s'y attaquait régulièrement au mari trompé, qui s'acharnait à se venger de son épouse infidèle. Louis Semitte ne s'en montrait pas moins tenace dans ses tentatives de faire exécuter en bonne et due forme les sentences rendues par les juges. Peu avant la parution de la Septième Promenade de Le Noble, Semitte, qui savait que sa femme détenue était enceinte, demanda et obtint un arrêt du Parlement interdisant de noter sur le registre des baptêmes le nom du père de l'enfant attendu. Nous ignorons les raisons précises qui motivèrent l'épicier à faire cette demande. Peut-être voulait-il simplement utiliser cette interdiction à l'appui d'une nouvelle accusation d'adultère contre sa femme. Il est également possible qu'il ait déjà flairé le tour que le couple Perreau-Le Noble allait essayer de lui jouer. La naissance de ce troisième enfant, une fille nommée Anne-Catherine, et l'interdiction de mettre le nom de son père sur le registre des baptêmes, donnèrent en tout cas une nouvelle tournure au procès; en juin 1699, Marie-Gabrielle Perreau fit une requête au Parlement pour faire reconnaître les deux petites comme 'vraies et légitimes filles' de Louis Semitte. Auparavant il n'avait jamais été question d'attribuer à Semitte les enfants illégitimes que Perreau avait eus avec Le Noble. Ils avaient été déclarés sous des noms de parents inventés. Mais cette fois-ci, il n’y avait pas moyen 
de dissimuler le nouveau-né. Ce bébé et sa sœur aînée, dont Semitte venait de découvrir l'existence, pouvaient donc être considérés comme des preuves indubitables de la relation adultère entre Marie-Gabrielle Perreau et Eustache Le Noble. La seule solution était de faire passer les deux filles comme les fruits d'une réconciliation entre Perreau et Semitte et c'est exactement ce que les deux amants essayèrent de faire. Le livre imaginaire des Quatre filles Aymon ou les Enfans trouvez sur notre catalogue satirique forme en quelque sorte le prélude à ce changement de stratégie de la part des deux amants: il n'y a pas de doute que les deux jolies nymphes dans le titre de ce livre imaginaire sont les deux filles de Perreau et de Le Noble, dont l'une se trouvait à l'Hôpital des Enfants-Trouvés. ${ }^{17}$ Et Cornificus, père présumé des deux petites filles, est bel et bien le mari infortuné de la dame Perreau. Ce sobriquet a été choisi pour son association avec le mot 'corne', symbole du mari trompé. Le jeu de mots est encore renforcé par l'emploi de l'expression 'corne d'abondance'. Étant donné que Saint Yves était le patron de toutes les professions de justice et de droit, et plus spécialement des avocats, le 'Maître Yves aussi cornu que lui' doit désigner l'avocat de Semitte, maître Aligier. ${ }^{18}$

\section{Encore des procès}

Un autre titre connu de la Bibliothèque bleue troyenne apparaît trois fois sur la liste de Mercure, chaque fois sous une forme différente. C'est l'Histoire de Pierre de Provence et de la belle Maguelonne, également connue sous le titre abrégé de Pierre de Provence ou sous celui de La belle Maguelonne. Est-ce par manque d'inspiration que Le Noble a utilisé trois fois le même livre de base ou la raison de ce choix réside-t-elle dans le fait que ce titre est un des rares livres d'amour dans le corpus bleu? Nous ne saurions le dire avec certitude, mais la première allusion à ce 'livre bleu' avait aisément pu être remplacée par un autre titre du répertoire des Oudot, à savoir La belle Hélène de Constantinople. Voici les termes dans lesquels ce premier titre est conçu : La Belle Maguelone, ou l'art de peindre, [...] Ouvrage [...] où l'on enseigne de quelle manière les vieux sepulchres sont reblanchis, \& comment on récrépit délicatement de vieilles murailles, en y apliquant de fortes couches de céruses \& de vermillon ; moyen [...] pour avoir

\footnotetext{
${ }^{17}$ Quant au premier-né, on le passait sous silence, parce que Marie-Gabrielle avait toujours nié son existence et qu'elle pensait que Semitte n'était pas au courant de sa naissance en 1694. L'enfant était d'ailleurs décédé à l'âge de quinze mois.

${ }^{18}$ Les écrits de Le Noble fourmillent de railleries à son adresse. Nous préparons un article sur l'affaire SemittePerreau-Le Noble sous le titre "Louis Semitte, mari trompé". Ce texte doit constituer la contrepartie historique du roman populaire, mais pas tout à fait fiable, de Pierre Darmon, intitulé Gabrielle Perreau, femme adultère (Paris: 1981).
} 
toûjours deux visages l'un sur l'autre, le Domestique fané dessous, \& le postiche brillant dessus. Jouant sur l'adjectif 'belle' dans le titre de l'histoire médiévale, Le Noble tourne l'histoire de La belle Maguelonne en un guide de maquillage. La vieille qui se farde pour rehausser ses chances de succès sur le marché des relations amoureuses est un thème récurrent dans les écrits satiriques de Le Noble, de même que celui des fausses apparences. À notre avis, il faut cependant interpréter ce titre fictif à la lumière des mutations sociales à la fin du XVII ${ }^{\mathrm{e}}$ siècle. Le fait que l'auteur parle ici d'un 'domestique fané' qui se cache sous le masque d'un homme flamboyant nous rappelle les observations de certains auteurs de l'époque sur la rapide ascension sociale d'anciens laquais et d'autres gens issus d'un milieu peu privilégié. Plusieurs hommes, que l'on savait d'origine modeste, avaient su amasser des fortunes considérables dans le commerce et dans la finance et étaient devenus des hommes puissants. Certains d'entre eux étaient même parvenus à se faire anoblir. En tant que membre d'une vieille famille noble, notre auteur partageait probablement le point de vue de ceux qui considéraient ces évolutions d'un mauvais œil. Il s'est en tout cas moqué plusieurs fois de la basse extraction de certains de ces nouveaux riches. Comme nous le verrons, notre catalogue satirique en contient un autre exemple plus élaboré.

La deuxième fois que l'histoire de Pierre de Provence apparaît sur le catalogue, c'est sous le titre de Pierre de Provence, ou l'homme aux deux femmes. [...] contenant une Instruction fort utile à ceux qui grace à leur argent bien ou mal acquis, veulent impunément se donner publiquement deux femmes tout à la fois [...]. Le tout composé \& mis en lumiere par Jean de Nivelle, \& dédié au Sieur de Belalgoisil. Ici le vieux roman chevaleresque a été choisi comme référence du fait qu'il raconte une histoire d'amour. La clé du titre se trouve dans la dédicace au Sieur de Belalgoisil: ce nom s'avère une allusion cryptique au nom du protagoniste d'un procès qui a fait presque autant de bruit à l'époque que l'affaire Semitte. Il s'agit du cas de Vincent Beausergent, un homme de naissance très modeste qui avait accumulé une fortune considérable et qui avait été accusé de bigamie par son amour de jeunesse. ${ }^{19}$ Quant au nom de Jean de Nivelle, cité comme auteur de ce livre fictif, on l'utilisait au XVII ${ }^{\mathrm{e}}$ siècle pour désigner un homme lâche et peu fiable. ${ }^{20}$ Mais Nivelle était aussi le nom de l'avocat d'un autre bigame

\footnotetext{
${ }^{19}$ Cf. Gayot de Pitaval François, Causes célèbres et intéressantes avec les jugemens qui les ont décidées. Nouvelle édition [...], tome III (Paris, Théodore Le Gras: 1750) 136-267.

${ }^{20}$ Cf. Corneille Thomas, Dictionnaire universel, géographique et historique, tome II (Paris, Jean Baptiste Coignard: 1708) 819 et Loret Jean, La Muze historique, éd. Ch.-L. Livet, tome III (Paris, P. Daffis: 1877) 204.
} 
impliqué vers la même époque dans un procès encore plus retentissant que celui de Beausergent. C'est justement ce procès où Louis Nivelle représentait un homme bigame qui semble avoir donné lieu au troisième titre du catalogue s'inspirant de l'histoire de Pierre de Provence et de la belle Maguelonne, à savoir Pierre de Provence, Histoire curieuse d'un homme tué qui se porte bien, remarié sans être veuf, \& père sans y penser. Le protagoniste de cette affaire était Louis de La Pivardière, seigneur de Narbonne. Dans le cadre de ses obligations professionnelles, celui-ci s'absentait régulièrement pour de longues périodes. En août 1697, il retourna à son château, mais le lendemain de son retour il fut porté disparu. L'épouse et son amant furent soupçonnés de meurtre et jetés en prison. Mais la vérité était que ce noble seigneur menait une double vie: il avait établi une famille à Auxerre et ne revenait de temps en temps à Narbonne que pour garder les apparences et pour chercher de l'argent. La Pivardière n'osait pas se montrer lors du procès, parce qu'il craignait d'être condamné pour bigamie. Et quand il fit finalement surface, son identité fut mise en question. Dans sa Cinquième Promenade, Le Noble avait déjà utilisé les curieux faits se rapportant à cette histoire qui passait pour un exemple criant de la stupidité des hommes de justice de province. ${ }^{21}$

Les sous-entendus dans le titre suivant, Le nouveau Jean de Paris, restent pour le moment obscurs, mais l'intention générale de Le Noble paraît claire. Le très long sous-titre explique que ce livre donne une Relation curieuse des magnificences, train [...] table \& maisons du Comte de Nevola, avec une Description des moyens [...] par lesquels on peut [...] du derriere d'un carosse sauter dedans. Et pour convaincre de l'infaillibilité de ces moyens, on y décrit la vie de vingt-quatre Exlaquais, dont le moindre joüit actuellement de plus de quarante mille livres de rente, le tout dédié au Sieur de Valsibour. Cela nous permet de constater que dans ce cas-ci, il y a effectivement un lien entre le titre fictif composé par Le Noble et le contenu du roman médiéval de Jean de Paris, dont les éditeurs de la Bibliothèque bleue multipliaient encore les éditions à la fin du XVII ${ }^{\mathrm{e}}$ siècle. Le vieux roman chevaleresque décrit amplement le magnifique train accompagnant le protagoniste lors d'un voyage en Espagne qui constitue l'intrigue principale de l'histoire. Le souvenir de cet équipage somptueux de Jean de Paris était bien vivant dans la France du Grand Siècle, entre autres dans la locution proverbiale 'mener un train de Jean de Paris', et c'est ce souvenir que Le Noble met ici à profit pour soulever le point de l'opulence des nouveaux riches qui étaient en train de gravir les échelons de la société. C'est

\footnotetext{
${ }^{21}$ Voir L'Avanture du mandarin OK-Piva dans Les Oeuvres de $M^{r}$ Le Noble, tome XII (Paris, Pierre Ribou: 1718) 359-371. Cf. aussi Gayot de Pitaval, Causes célèbres 1-135.
} 
un sujet qui revient souvent dans ses écrits, notamment dans un contexte où il fulmine contre les moyens scandaleux dont les hommes de finance se servaient pour s'enrichir. ${ }^{22}$ La satire de la malhonnêteté, la grossièreté et la vanité des riches et puissants fermiers, ou partisans comme on les appelait alors, était en effet un thème bien répandu dans la littérature de l'époque. Un des éléments de l'image stéréotypée et probablement peu réelle que l'on y présentait de ces gens était leur naissance 'ignoble' et leur ancienne condition servile. Selon Daniel Dessert, cette représentation des riches financiers comme d'anciens laquais, que l'on trouve au sein de notre catalogue satirique dans les titres de Jean de Paris et de La belle Maguelonne, réapparaît comme un 'leitmotiv obsédant' dans les témoignages du XVII ${ }^{\mathrm{e}}$ siècle finissant. Elle aurait donné lieu à un véritable 'mythe du laquais financier'. ${ }^{23}$ Il n'est pas impensable qu'en dotant le dédicataire imaginaire du Nouveau Jean de Paris du nom du 'Sieur de Valsibour', Le Noble vise un des financiers les plus puissants du début du XVIII ${ }^{\mathrm{e}}$ siècle, à savoir Paul Poisson, sieur de Bourvallais, dont l'opulence était proverbiale. Avant de devenir le protégé du contrôleur général des finances, le Comte de Pontchartrain, Paul Poisson aurait en effet servi comme laquais chez un autre homme d'argent. ${ }^{24}$ Quant au 'comte de Nevola', nous n'avons pas encore trouvé d'explication satisfaisante pour ce nom.

Le titre qui suit touche plus ou moins à ce même sujet des richesses faussement acquises par des hommes de finance. C'est Huon de Bordeaux ou le Gigès de la Douane, par lequel s'expliquent naïvement tous les tours de souplesses dont se servent les Publicains pour tromper leur Maître, pour se tromper les uns les autres, et pour tromper le public. L'histoire populaire de Huon de Bordeaux telle qu'elle était diffusée dans la Bibliothèque bleue de l'époque était un remaniement d'une chanson de geste médiévale où le merveilleux jouait un rôle prépondérant. Nous ne savons pas si c'est le contenu de l'ancienne chanson de geste ou seulement le nom du protagoniste qui a servi dans ce cas comme référence à Le Noble. Il y a en tout cas un point commun entre l'histoire de Huon de Bordeaux et le mythe classique de Gygès évoqué dans le sous-titre de ce livre imaginaire : leurs protagonistes possédaient tous les deux un anneau magique qui leur donnait la faculté de se rendre invisible. Selon le mythe de l'anneau de Gygès

\footnotetext{
${ }^{22}$ Cf. par exemple Les Oeuvres de $M^{r}$ Le Noble, tome IV 103 et 150-189.

${ }^{23}$ Cf. Dessert D., Argent, pouvoir et société au Grand Siècle (Paris: 1984) 98-109.

${ }^{24} \mathrm{Cf}$. sur ce personnage dont le nom ou les initiales figurent dans plusieurs textes satiriques de l'époque, Challe Robert, Mémoires, Correspondance complète, Rapports sur l'Acadie et autres pièces, éd. F. Deloffre (Geneva: 1996) $298-311$.
} 
dans la version rapportée par Platon, le berger Gygès trouva un jour un anneau, et après avoir découvert son pouvoir magique, il s'en servit pour s'introduire dans le palais du roi de Lydie afin de séduire la reine, d'assassiner le roi et de prendre lui-même le pouvoir du pays. Le Noble compare donc l'usage que fit Gygès de son anneau magique aux astuces qu'utilisent les douaniers pour acquérir argent et pouvoir. Le mot 'publicains' qu'il emploie pour désigner ces gens a été choisi par analogie avec les malhonnêtes receveurs des revenus publics romains cités dans la Bible.

À côté de ces huit titres fictifs inspirés par des anciens romans de chevalerie, notre catalogue satirique cite deux autres titres qui ne semblent pas faire allusion à des livres qui existaient réellement dans la Bibliothèque bleue. Le premier est Joconde la Dévergondée, ou le Secret des coulisses révélé; Histoire qui contient quarante deux avantures recréatives [...] dont le but est toûjours de plumer des oisons, pour faire de leur Duvet de bons [...] coussins, aux fauteüils \& aux canapez des opératrices. Avec ce titre, Le Noble revient de nouveau à un sujet qu'il avait déjà abordé plusieurs fois dans ses ouvrages: celui de la cupidité des 'opératrices', c'est-à-dire des filles de l'opéra, qui ne répondent aux avances de leurs admirateurs que pour les plumer. L'image d'un oiseau perdant des plumes apparaît régulièrement dans ce contexte. ${ }^{25}$ Le mot 'oison' avait alors déjà la connotation de 'personne crédule, facile à mener'. Si l'on peut croire Jean-Nicolas de Tralage, les seigneurs de la Cour et les riches partisans du XVII ${ }^{\mathrm{e}}$ siècle finissant s'empressaient auprès des filles de l'opéra. Avoir une fille de l'opéra comme maîtresse était alors de bon ton. Et les belles n'hésitaient évidemment pas à en profiter. Le recueil de Tralage recense plusieurs chansons populaires à propos du prix des faveurs de certaines dames. ${ }^{26}$ Le nom de Joconde dans le titre de cet ouvrage fictif réfère probablement à un personnage du Roland furieux, poème chevaleresque de l'Arioste très goûté par le public mondain du Grand Siècle, mais vilipendé par certains critiques, qui le mettaient sur le même rang que les vieux romans de chevalerie français diffusés par les éditeurs de la Bibliothèque

\footnotetext{
${ }^{25}$ Cf. par exemple Le Noble Eustache, La Grotte des fables (Paris, Martin et Georges Jouvencel: 1696) Troisième fable: De l'Oiseleur, des oiseaux \& du miroir; Quatrième fable: Du serpent \& de l'enclume 2829; Huitiéme fable: De la Chatte changée en fille 4.

${ }^{26}$ Tralage Jean-Nicolas de, Notes et documents sur l'histoire des théâtres de Paris au XVII siècle, éd. Le Bibliophile Jacob (Paris: 1880). Cf. aussi la Description de la vie et mours, de l'exercice et l'état des filles de l'opéra, manuscrit datant des années 1690 et attribué à l'abbé de Vassetz. Ce texte a été publié dans Ladvocat Lois François., Lettres sur l'opéra à l'abbé Dubos, éd. J. de La Gorce (Paris: 1993).
} 
bleue. Joconde était un très bel homme qui, après avoir été trompé par sa bien-aimée, décida de se venger du sexe faible en devenant un coureur de jupons. ${ }^{27}$

Le titre du dernier livre qui reste de la liste imaginaire de Le Noble, à savoir Alain Chartier ou le Bureau d'adresse, rentre très bien dans la catégorie des 'livres bleus' troyens. Le nom d'Alain Chartier suggère une origine médiévale qui était effectivement typique pour une partie des textes appartenant à la Bibliothèque bleue. Le genre aussi est bien choisi. Le Bureau d'adresse était un bureau de renseignements et les livres pratiques, comme les almanachs, les livres de recettes et les modèles d'art épistolaire, étaient bien représentés sur le catalogue des Oudot et de leurs confrères. La suite du titre révèle cependant que le nom d'Alain Chartier est un jeu de mots: au XVII ${ }^{\mathrm{e}}$ siècle, la dénotation du mot 'chartier' était : 'celui qui mène une charrette' ou 'conducteur de carrosse'. Aussi ce livre imaginaire porte-t-il sur des carrosses. Le sous-titre dit: ouvrage [...] où l'on explique [...] l'utilité [...] des carosses de commodité établis de tous les côtez pour conduire en toute diligence aux Penitentes les vieilles veuves qui épousent de jeunes maris; les vieillars qui épousent de jeunes femmes, aux Invalides. Les Joueurs à l'Hôpital, les Poëtes à la Friperie, les Plaideurs à la Valée de misere, les Amans aux Incurables, les Jaloux aux Petites maisons, la plûpart des Auteurs à la Pitié, les Financiers à Montorgueil, les petits Maîtres à la Monoye, \& presque tous les hommes au S. Esprit. Le phénomène des carrosses à louage, dont on disait dans les documents officiels qu'ils avaient été 'établis pour la commodité publique', n'était pas nouveau en 1699. On les trouvait un peu partout à Paris, non seulement dans des remises, mais aussi sur les places et les carrefours. Les ordonnances concernant leur exploitation et les confrontations entre ceux qui en détenaient le privilège et leurs concurrents faisaient cependant encore souvent les frais de la conversation vers le tournant du XVIII ${ }^{\mathrm{e}}$ siècle. Dans l'énumération rabelaisienne donnée dans ce titre, Le Noble rattache de manière ludique quelques types sociaux dont il parle régulièrement dans ses publications satiriques à un certain nombre d'institutions parisiennes d'assistance et de répression sociale et à quelques autres endroits bien connus des Parisiens de l'époque. Les trois thèmes principaux de son œuvre morale, qui sont déjà largement présents dans notre catalogue satirique, à savoir l'argent, l'amour' et les procès, se trouvent ici réunis en un seul titre. On pourrait ranger les joueurs, les poètes, les auteurs, les petits maîtres et les financiers sous le dénominateur thématique commun de la bourse. Les quatre premières catégories manquent de

\footnotetext{
${ }^{27}$ Dans la littérature du XVII siècle, nous n'avons pas trouvé d'exemples de l'emploi du nom de 'Joconde' pour désigner la célèbre peinture de Léonardo Da Vinci.
} 
ressources et se retrouvent respectivement à l'Hôpital général (lieu de renfermement de mendiants, vagabonds et autres pauvres), chez des marchands fripiers, à la Pitié, c'est-à-dire au mont-de-piété, et à la Monnaie. Montorgueil, le nom significatif du lieu où sont conduits les financiers prétentieux qui roulent sur l'or, était le nom d'une petite colline à Paris qui avait donné son nom à une des rues de la capitale. Quant aux groupes associés au thème de l'amour - les amants, les jaloux, les personnes âgées ayant épousé une jeune personne -, ils finissent soit dans une maison religieuse soit dans une maison de fous. Seuls ceux qui sont envoyés aux Invalides échappent en quelque sorte à cette règle. Le nom de la destination des plaideurs est aussi significatif que celui du terminus des carrosses dans lesquels se trouvent les financiers. Tout comme Montorgueil, la Vallée de Misère est une appellation que l'on utilisait effectivement pour indiquer un endroit réel, à savoir l'actuel Quai de la Mégisserie où se trouvait un marché de volailles et de gibier. ${ }^{28}$ Le quartier était habité par des bouchers et l'odeur qui se dégageait de ce lieu était quasi insupportable. Nous ne savons pas quel endroit Le Noble désigne dans la dernière partie du titre, où il est question d'hommes conduits au S. Esprit. Il y avait l'hôpital du Saint-Esprit, mais c'était un orphelinat. Peut-être l'auteur réfère-t-il à une boutique ou à un cabaret qui avait pour enseigne le Saint-Esprit?

\section{Conclusions}

Après la présentation de cette dizaine de titres, la nouvelle du Colporteur de Proserpine se termine assez abruptement avec la décision des quatre amis de remettre au lendemain la lecture du reste du mémoire sur Barbin. Cela n'empêche pas que ce catalogue est l'exemple le plus élaboré d'une liste de livres imaginaires bâtie sur des titres d'anciens romans de chevalerie ou de livrets de la Bibliothèque bleue que nous ayons pu trouver dans la littérature du XVII ${ }^{\mathrm{e}}$ siècle. Presque toutes les autres listes de livres imaginaires de l'époque qui donnent la part belle aux vieux romans et aux 'livres bleus' se contentent d'en citer les titres originaux. Parfois ces titres sont accompagnés d'une courte allusion ludique au contenu des histoires concernées, mais il semble que la simple énumération des titres de ces romans familiers d'une époque révolue et des livrets qui faisaient les délices du public modeste de la Bibliothèque bleue suffisait alors pour faire rire. Certes, on peut trouver quelques exceptions, comme par exemple le Roman bourgeois de Furetière qui contient un catalogue avec des titres imaginaires basés sur le roman-

\footnotetext{
${ }^{28}$ L'expression est attestée dans la troisième édition du dictionnaire de Furetière. Cf. Furetière Antoine, Dictionnaire universel [...], tome III, (Rotterdam, Reinier Leers: 1708) n.p.
} 
fleuve des Amadis et sur Ogier le Danois, mais ce sont des cas isolés qui ne font pas partie d'une liste de vieux romans ou de livres populaires. ${ }^{29}$ Comparé à la plupart des bibliothèques et catalogues de livres imaginaires que nous avons eus sous les yeux dans le cadre de nos recherches sur les vieux romans, le catalogue de Le Noble est relativement riche et plus proche du modèle de Rabelais. Le Noble semble en effet avoir admiré Rabelais, qu'il cite à plusieurs reprises dans ses œuvres. Son très petit catalogue du Colporteur de Proserpine a beau être un travail fait à la va-vite par un plumitif poursuivi par la justice et dont la bibliothèque de référence se trouvait probablement uniquement dans sa tête, il fait plus honneur à l'inventeur du genre que maint autre catalogue satirique du XVII ${ }^{\mathrm{e}}$ siècle.

Dans ce contexte, il n'est pas moins intéressant de noter que, contrairement à la majeure partie des listes de livres fictifs publiées au XVII ${ }^{\mathrm{e}}$ et au début du XVIII ${ }^{\mathrm{e}}$ siècle, la liste de Le Noble a paru dans une œuvre dont les contours génériques sont difficiles à établir. Il ne s'agit pas de l'évocation du contenu d'une bibliothèque ou de l'inventaire d'une librairie, d'un étalage de bouquiniste ou d'un ballot de colporteur dans le cadre narratif et fictif d'un roman, d'un poème ou d'une description burlesque de telle ou telle ville européenne. Ce n'est pas non plus un catalogue d'une collection imaginaire sous la forme d'un pamphlet ou d'une autre publication isolée, comme La Bibliothèque du roi Guillemot. Si, par leur nombre réduit de pages, leur forme éphémère et leur apparition périodique, Les Promenades de Le Noble sont à rapprocher des pasquinades et des journaux, le contenu du Colporteur de Proserpine relève à la fois du genre de l'entretien, du dialogue des morts, de la nouvelle, de la fable et de la liste de livres imaginaires. Et bien qu'à l'instar de nombre d'autres listes de livres du Grand Siècle qui citent des romans chevaleresques et des livres populaires, le catalogue de Le Noble vise à stigmatiser tout un corpus de livres et à critiquer l'inculture et la superficialité littéraire des lecteurs mondains, ses enjeux littéraires semblent être éclipsés par un autre objectif de l'auteur, à savoir la satire morale de la société contemporaine. De ce point de vue on pourrait mettre $L e$ Colporteur de Proserpine en rapport avec Les Caractères de Jean de La Bruyère, ouvrage qui récoltait un grand succès à l'époque. Dans ses observations sur les mœurs du siècle, La Bruyère touche aux mêmes thèmes que Le Noble. Les deux hommes semblent avoir partagé les mêmes pensées à propos de certains de leurs contemporains qu'ils présentent tous les deux sous des noms inventés ou raccourcis. Si l'intérêt des portraits satiriques qu'ils brossent est universel, les lecteurs contemporains pouvaient deviner l'identité de ceux qui en avaient été les modèles.

\footnotetext{
${ }^{29}$ Furetière, Le Roman bourgeois 279 et 282.
} 
La suggestion de Philippe Hourcade disant que '[s]'il était possible d'identifier tous les noms de fantaisie répandus' dans l'œuvre de Le Noble, 'se révéleraient peut-être à nos yeux les éléments épars d'une véritable chronique du temps' s'est en tout cas avérée être tout à fait justifiée. ${ }^{30}$ Il existe d'ailleurs un autre catalogue fictif de la fin du XVII ${ }^{\mathrm{e}}$ siècle qui se lit aussi comme un livre à clé donnant à mots couverts le récit de nouvelles ou rumeurs à propos des gens en vue. C'est le Catalogue de livres nouveaux à Paris, texte anonyme datant de $1693 .{ }^{31}$

La clé pour comprendre les allusions satiriques et ludiques réside parfois dans les noms des auteurs ou des dédicataires fictifs, mais aussi dans d'autres publications de Le Noble. Il est frappant de constater que Le Colporteur de Proserpine contient tous les grands thèmes de l'œuvre moraliste de l'auteur, que l'on pourrait appeler aussi ses chevaux de bataille, des attaques personnelles contre Louis Semitte ou Claude Barbin à la critique des mœurs des filles de l'opéra, des mauvais conseils des procureurs aux malversations des fermiers généraux. En ce sens, le catalogue de Le Noble est un bel exemple d'intertextualité personnelle. Soulignons dans ce contexte encore que la place prépondérante attribuée à des sujets empruntés au domaine juridique s'explique par la formation de Le Noble et par le grand nombre de procès dans lesquels il a été impliqué lui-même. ${ }^{32}$

La liste de livres imaginaires composée par le Noble reflète donc d'abord les préoccupations personnelles de l'auteur, homme de lettres, moraliste et homme de loi. Mais Le Noble s'est en même temps fait le porte-parole des sentiments et des curiosités qui existaient au sein de la société de l'époque. On pourrait peut-être même dire qu'avec son insistance sur les faits divers, son intérêt pour la vie des grands et la présence d'une historiette à la mode, $L e$ Colporteur de Proserpine a plus en commun avec Le Mercure galant de Barbin que Le Noble n'aurait jamais voulu l'admettre.

\footnotetext{
${ }^{30}$ Hourcade, Entre Pic et Rétif 388

${ }^{31}$ Ce catalogue est contenu dans le Recueil Tralage (Paris, BnF, Ars. ms. 6543, fol. 42). Parmi les 90 entrées de la liste il n'y a qu'un seul roman de chevalerie, Les Amours de la belle Magdelaine et de Pierre de Provence, titre qui fait allusion aux amours présumées de l'archevêque de Paris et de Madame de Lesdiguières.

${ }^{32}$ En dépit de toutes ses invectives contre les plaideurs et leurs procureurs, Le Noble n'a pas hésité à épuiser luimême toutes les possibilités que lui offrait le système. Procéder en justice et rester au courant des affaires en cours était chez lui comme une seconde nature.
} 
Bibliographie sélective

Blom H., «Vieux Romans » et «Grand Siècle »; éditions et réceptions de la littérature chevaleresque médiévale dans la France du dix-septième siècle (unpublished $\mathrm{PhD}$ dissertation Utrecht University: 2012).

DESSERT D., Argent, pouvoir et société au Grand Siècle (Paris: 1984).

GAYOT DE PitAVAL FRANÇOIS, Causes célèbres et intéressantes avec les jugemens qui les ont décidées. Nouvelle édition [...], tome III (Paris, Theodore Le Gras: 1750).

[GILlet FrançoIS-PIERRE,] Plaidoyers et autres æuvres de François-Pierre Gillet. Avocat au Parlement. Nouvelle édition, 2 vols (Paris, Gabriel Martin: 1718).

Goulemot J.M., "En guise de conclusion: les bibliothèques imaginaires (fictions romanesques et utopies)" in JOLLY C. (éd.), Histoire des bibliothèques françaises, tome II (Paris: 1988) 500-511.

GRANDE N., "Claude Barbin, un libraire pour dames?”, Revue de la BNF 39 (2011) 22-27.

Hourcade PH., Entre Pic et Rétif: Eustache le Noble (1632-1711) (Paris: 1990).

[Le Noble Eustache,] Les Oeuvres de $M^{r}$ Le Noble, 19 vols (Paris, Pierre Ribou: 1718).

MANDROu R., De la culture populaire aux $17^{e}$ et $18^{e}$ siècles, $3^{\mathrm{e}}$ éd. (Paris: 1985).

REED G.E., Claude Barbin, libraire de Paris sous le règne de Louis XIV (Genève - Paris: 1974).

SGARD J. (éd.), Dictionnaire des journaux 1600-1789 (Oxford: 1991). Édition électronique revue, corrigée et augmentée.

SGARD J. (éd.), Dictionnaire des journalistes 1600-1789 (Oxford: 1999). Édition électronique revue, corrigée et augmentée.

SIMONIN M., "La réputation des romans de chevalerie selon quelques listes de livres $\left(\mathrm{XVI}^{\mathrm{e}}\right.$ XVII ${ }^{\mathrm{e}}$ siècles)", in Mélanges de langue et littérature françaises du Moyen Âge et de la Renaissance offerts à Monsieur Charles Foulon [...], tome I (Rennes: 1980) 363-369. 


\section{Extrait du Colporteur de Proserpine}

Le Noble Eustache, Les CEuvres de Mr Le Noble, tome XII (Paris, Pierre Ribou: 1718) 427-430.

Mercure grand Maître des ceremonies des Enfers, \& qui fait de frequens voyages sur terre, voulut se charger de passer à Troyes chez Oudot, \& d'en raporter tous les plus beaux enfans bleus qu'il y verroit. Et voici la Liste de ceux que dès le premier voyage il lui [Barbin $\mathrm{HB}$ ] raporta.

Le nouveau Jean de Paris, ou la Relation curieuse des magnificences, train, équipage, table \& maisons du Comte de Nevola, avec une Description des moyens innocens par lesquels on peut avec promptitude $\&$ facilité du derriere d'un carosse sauter dedans. Et pour convaincre de l'infaillibilité de ces moyens, on y décrit la vie de vingt-quatre Exlaquais, dont le moindre joüit actuellement de plus de quarante mille livres de rente, le tout dédié au Sieur de Valsibour.

La Belle Maguelone, ou l'art de peindre, petit Ouvrage merveilleux où l'on enseigne de quelle maniere les vieux sepulchres sont reblanchis, \& comment on récrépit délicatement de vieilles murailles, en y apliquant de fortes couches de céruses \& de vermillon; moyen curieux pour avoir toûjours deux visages l'un sur l'autre, le Domestique fané dessous, \& le postiche brillant dessus.

Joconde la Dévergondée, ou le Secret des coulisses révélé; Histoire qui contient quarante deux avantures recréatives, toutes plus plaisantes les unes que les autres, \& dont le but est toûjours de plumer des oisons, pour faire de leur Duvet de bons \& doüillets coussins, aux fauteüils \& aux canapez des opératrices.

Pierre de Provence, ou l'homme aux deux femmes. Petit Livret, contenant une Instruction fort utile à ceux qui grace à leur argent bien ou mal acquis, veulent impunément se donner publiquement deux femmes tout à la fois, quoi que souvent la moitié d'une soit encore trop pour un homme tout entier. Le tout composé par Jean de Nivelle, \& dédié au Sieur de Belalgoisil.

Alain Chartier, ou le Bureau d'adresse, ouvrage excellent, où l'on explique d'une maniere élegante l'utilité admirable des carosses de commodité établis de tous les côtez pour conduire en toute diligence aux Penitentes les vieilles veuves qui épousent de jeunes maris ; les vieillars qui épousent de jeunes femmes, aux Invalides. Les Joueurs à l'Hôpital, les Poëtes à la Friperie, les Plaideurs à la Valée de misere, les Amans aux Incurables, les Jaloux aux Petites maisons, la plûpart des Auteurs à la Pitié, les Financiers à Montorgueil, les petits Maîtres à la Monoye, \& presque tous les hommes au S. Esprit.

Les quatre Filles Aymon, ou les Enfans trouvez. Petit Livre qui contient de serieuses réflexions, sur la merveilleuse \& sage conduite du fameux Cornificius, \& comment par les conseils d'un Maître Yves aussi cornu que lui, \& à force de remuer la corne d'abondance dont il est authentiquement pourvû, il a eu le bonheur d'en faire miraculeusement sortir deux jolies petites Nymphes qui lui ressemblent comme deux goutes d'eau.

Pierre de Provence, Histoire curieuse d'un homme tué qui se porte bien, remarié sans être veuf, \& pere sans y penser.

Huon de Bordeaux, ou le Gigès de la Douane, par lequel s'expliquent naïvement tous les tours de souplesses dont se servent les Publicains pour tromper leur Maître, pour se tromper les uns les autres, \& pour tromper le public.

Geofroi à la Grand dent, ou le Plaideur Béat. Livret dans lequel on voit le bon \& delectable usage que l'augure Theogon fait de ses revenus, qu'il employe à nourir charitablement tous les jours plus de cinquante bouches, tant d'Huissiers, Sergens, Procureurs \& Avocats, que de Greffiers, Secretaires \& Juges, le tout conformement à la pieuse destination des Fondateurs.

Il seroit trop ennuieux de vous expliquer tous les autres Livres les uns après les autres; $\&$ l'on peut juger de ceux-ci, que sa Boutique est du moins aussi curieusement fournie là-bas, qu'elle l'étoit sur terre.

Je veux seulement vous copier une petite Historiette qui en faisoit le principal ornement, \& qui fut le premier Livre dont il régala Proserpine. Ainsi laissant tout le reste, lisez cette avanture, \& je croi que vous ne la trouverez pas indigne de vous amuser un moment.

[Suit Melusine ou La Vieille Fardée] 